TRABAJOS ORIGINALES

Rev Obstet Ginecol Venez. 2021; 81 (4): 329-341.

https://doi.org/10.51288/00810406

\title{
Efectos adversos perinatales asociados a adiposidad materna durante la gestación
}

\author{
(D) Héctor J. Hernández A, ${ }^{1}$ (D) Joseph R. Hernández A, ${ }^{1}$ (D) Freddy Bello Rodríguez. ${ }^{2}$
}

\begin{abstract}
RESUMEN
Objetivo: Determinar efectos adversos perinatales asociados a adiposidad materna durante la gestación entre noviembre 2017 y octubre 2018, en los Servicio de Obstetricia y Ginecología en el Hospital Universitario: Dr. Jesús María Casal Ramos, Acarigua - Araure, Edo. Portuguesa.

Métodos: Estudio descriptivo, retrospectivo, transversal, con muestra conformada por 115 historias clínicas de gestantes con sobrepeso y obesidad.

Resultados: No se encontraron primigestas adolescentes precoces. Entre las 67 pacientes con sobrepeso y con obesidad tipo I, predominaron mujeres con 26-30 años (22/32,8 \% y 10/34,5\%), seguidas del grupo 21-25 (18/26,9\% y 8/27,6 \%). Las pacientes con obesidad tipo II y III tuvieron una distribución más homogénea. La ganancia del peso durante el embarazo fue menor de $10 \mathrm{Kg}$ en 85 pacientes (73,9\%). Predominaron los embarazos a término en todos los grupos $(91 \%, 82,2 \%$, $80 \%$ y 88,9\%). El parto fue predominantemente vaginal en las pacientes con sobrepeso y obesidad tipo I $(70,1 \%$ y $51,7 \%$ ), la cesárea fue más frecuente en la obesidad tipo II y III (60 \% y 55,6\%). Las complicaciones maternas más frecuentes en pacientes con sobrepeso fueron: infecciones vaginales $(25,4 \%)$, infecciones urinarias (14,9\%) y anemia $(14,9 \%)$. Entre las obesas hubo mayor frecuencia de trastorno hipertensivo del embarazo $(24,1 \%, 50 \%$ y 55,6\% en obesidad I, II y III, respectivamente). Entre las complicaciones neonatales, predominaron bajo peso y prematuridad.

Conclusiones: El sobrepeso y la obesidad son factores asociados a efectos adversos maternos y perinatales.
\end{abstract}

Palabras clave: Adiposidad, Efectos perinatales, Sobrepeso, Obesidad.

\section{SUMMARY}

Objective: To determine perinatal adverse effects associated with maternal adiposity during pregnancy between November 2017 and October 2018, in the Obstetrics and Gynecology Service at the University Hospital: Dr. Jesús Maria Casal Ramos, Acarigua - Araure, Edo. Portuguesa.

Methods: Descriptive, retrospective cross-sectional with a sample consisting of 115 clinical records of overweight and obese pregnant women.

Results: No early adolescent primigestas were found. Among the 67 overweight and type I obese patients, women aged 26-30 years (22/32.8\% and 10/34.5\%) predominated, followed by the 21-25 group (18/26.9\% and 8/27.6\%). Patients with type II and III obesity had a more homogeneous distribution. Weight gain during pregnancy was less than $10 \mathrm{~kg}$ in 85 patients (73.9\%). Full-term pregnancies predominated in all groups $(91 \%, 82.2 \%, 80 \%$ and $88.9 \%)$. Delivery was predominantly vaginal in overweight and obese patients type I (70.1\% and 51.7\%), cesarean section was more frequent in typi II and III obesity (60\% and $55.6 \%)$. The most frequent maternal complications in overweight patients were: vaginal infections $(25.4 \%)$, urinary tract infections $(14.9 \%)$ and anemia (14.9\%). Among obese women there was a higher frequency of hypertensive disorder of pregnancy $(24.1 \%, 50 \%$ and $55.6 \%$ in obesity I, II and III, respectively). Among the neonatal complications, low weight and prematurity predominated.

Conclusions: Overweight and obesity are factors related to maternal and perinatal adverse effects.

Key words: Adiposity, Perinatal effects, Overweight.

\section{INTRODUCCIÓN}

${ }^{1}$ Médico Residente. 2 Medico Jefe de Departamento Obstetricia y Ginecología, Hospital Universitario: Dr. Jesús María Casal Ramos, Acarigua. Correo de correspondencia: belfred63@gmail.com

Forma de citar este artículo: Hernández HJ, Hernández JR, Bello Rodríguez F. Efectos adversos perinatales asociados a adiposidad materna durante la gestación. Rev Obstet Ginecol Venez. 2021; 81(4):329-341. https://doi.org/10.51288/00810406
Las acumulaciones excesivas de tejido adiposo subyacen en el origen de diferentes enfermedades. El estado de adiposidad durante la gestación, expresado o no como sobrepeso u obesidad, constituye una amenaza 
para el bienestar materno fetal y su continuidad hacia el parto. En general, los depósitos adiposos femeninos son superiores y relevantes durante la gestación y después de ella, por su participación en la aparición de factores de riesgo materno infantil (1).

El sobrepeso, según la Organización Mundial de la Salud (OMS), se define como acumulación anormal o excesiva de grasa y la obesidad, es un desequilibrio energético entre calorías consumidas y gastadas causando acumulación excesiva de grasa perjudicial para la salud. El índice de masa corporal (IMC) es el indicador simple de la relación entre peso y talla que se utiliza frecuentemente para identificar sobrepeso en los adultos. Se considera sobrepeso cuando el IMC se encuentra entre $25-29,9 \mathrm{~kg} / \mathrm{m}^{2}$ y se define obesidad cuando el índice de masa corporal es igual o mayor a $30 \mathrm{~kg} / \mathrm{m}^{2}$, lo cual puede ser perjudicial para la salud. La obesidad se divide en tres tipos: obesidad tipo I o moderada, entre $30-34,9 \mathrm{~kg} / \mathrm{m}^{2}$, obesidad tipo II o grave, entre $35-39,9 \mathrm{~kg} / \mathrm{m}^{2}$ y obesidad tipo III o mórbida, cuando es mayor o igual a $40 \mathrm{~kg} / \mathrm{m}^{2}$ respectivamente $(2,3)$.

La obesidad favorece el desarrollo de una larga lista de afecciones entre las que destacan la diabetes mellitus tipo 2, las dislipidemias, la hipertensión arterial y diversos tipos de cánceres (mama, endometrio, colon, esófago, páncreas, etcétera). La obesidad en sí, y todas las comorbilidades que genera, aumentan la mortalidad de manera significativa, estimándose que la reducción de la esperanza de vida en estos sujetos es de por lo menos cinco años, en mujeres jóvenes no solo las predispone a enfrentar una mayor prevalencia de enfermedades crónicas, también incrementa su riesgo de infertilidad, el desarrollo de complicaciones en el embarazo y las alteraciones en el desarrollo fetal. Su presencia condiciona tantas complicaciones médicas o psicológicas, alteraciones neonatales, obstétricas, congénitas, cambios en el estado metabólico durante el embarazo con expresión en el posterior estado de salud de la mujer y desarrollo de sus hijos $(4,5)$.
La ganancia de peso óptima en embarazadas con peso preconcepcional normal fluctúa, en la mayoría de los estudios, entre 11 y 16 kilogramos. Sin embargo, depende en gran medida de su talla. Una ganancia de peso cercana a los 16 kilogramos en mujeres bajas, con tallas menores a $150 \mathrm{~cm}$, puede aumentar el riesgo de desproporción céfalo-pélvica. A la inversa, recomendar valores cercanos a 11 kilogramos en madres con una talla mayor de $160 \mathrm{~cm}$ puede aumentar el riesgo de desnutrición intrauterina (6).

Un índice de masa corporal pregestacional mayor de 30 se asocia a un incremento en la posibilidad de desarrollar complicaciones hipertensivas (el riesgo de preeclampsia es 3 veces mayor), diabetes gestacional (riesgo 4 veces mayor) y tromboembolias (constituyen la mayor causa de muerte materna) durante el embarazo. Debido a las complicaciones mencionadas, en muchas ocasiones debe inducirse el parto o realizarse una operación cesárea en las semanas 34 a 37 de gestación, dependiendo de la gravedad de la enfermedad, lo que incrementa aún más la posibilidad de complicaciones neonatales (7). Cuanto mayor es la obesidad, mayor es la posibilidad de que se deba realizar una cesárea anticipada, siendo la proporción de cesáreas de 2:1 entre las obesas en relación con las que no lo son. Se ha indicado que, por cada $5 \mathrm{~kg}$ de aumento de peso durante el embarazo, se incrementa $13 \%$ el riesgo de tener que realizar una cesárea (8).

El sobrepeso y la obesidad condicionan las mayores complicaciones del embarazo en la actualidad, tanto en países en vías de desarrollo como en países desarrollados. La obesidad presente antes del embarazo es un factor de riesgo independiente para complicaciones obstétricas como parto pretérmino, preeclampsia, macrosomía fetal, retraso del crecimiento intrauterino, traumatismo neonatal o hipoglucemia neonatal $(9,10)$.

Los niños nacidos de madres con sobrepeso y obesidad tienen mayor porcentaje de macrosomía que las de 
madres con estado nutricional normal lo que sugiere que sobrepeso y obesidad materna pueden ocasionar riesgo significativo para las nuevas generaciones con compromisos metabólicos desde etapas tan tempranas de la vida y muestra la importancia del periodo perinatal para la prevención de enfermedades crónicas no transmisibles $(1,11)$.

Las gestantes con sobrepeso y obesidad tienen riesgo alrededor de 2 a 6 veces más de padecer de diabetes gestacional que la embarazada clasificada con peso normal $(12$ - 14). El riesgo de sufrir preeclampsiaeclampsia es aún más elevado, también se eleva el riesgo de parto pretérmino, de requerir cesárea y de presentar complicaciones posoperatorias y macrosomía fetal, contribuyendo al incremento de la morbimortalidad perinatal. Durante el embarazo, la ganancia excesiva de peso es un factor negativo que implica complicaciones para la embarazada, feto y neonato; los embarazos de estas pacientes deben ser catalogados como embarazos de alto riesgo $(15,16)$.

Por lo formulado, se considera necesario describir los efectos adversos perinatales presentes en gestantes con sobrepeso y obesidad. El presente estudio se realizó con el objetivo de determinar tales efectos adversos perinatales asociados a adiposidad materna durante la gestación.

\section{MÉTODOS}

Se realizó un estudio descriptivo, retrospectivo, transversal, sobre la base de las historias clínicas de todas las gestantes ingresadas en los diferentes Servicios de Ginecología y Obstetricia del Hospital Central Universitario Dr. Jesús María Casal Ramos, Acarigua-Araure, Edo. Portuguesa (Maternidad, Sala de Parto, Alto Riesgo Obstétrico), durante el periodo 01 noviembre de 2017 - 30 de octubre de 2018. Se incluyeron 115 gestantes con gestación simple y con IMC mayor o igual de $25 \mathrm{~kg} / \mathrm{m}^{2}$. Se excluyeron aquellas cuyas historias clínicas no estuvieran disponibles, o la información requerida fuera incompleta.

La información se recolectó de las historias clínicas y se llenó una encuesta clínica estructurada en 5 segmentos en los cuales se incluyó: identificación, antecedentes personales y obstétricos, comorbilidades asociadas, complicaciones maternas y neonatales presentadas, evolución perinatal precoz. Los datos se presentaron asociándolos al IMC.

Posteriormente todos los datos recolectados se analizaron con el paquete estadístico para Windows SPSS 22, mediante frecuencias absolutas, relativas, medidas de tendencia central y de dispersión.

\section{RESULTADOS}

En el Departamento de Historias Médicas, Estadísticas y Archivo del Hospital Universitario Dr. "Jesús María Casal Ramos", se realizó la revisión de 5200 historias clínicas de gestantes correspondientes al periodo establecido. Se incluyeron 115 casos que llenaron los criterios establecidos. La clasificación según el IMC se observa en el gráfico 1 . Hubo 67 pacientes con sobrepeso (58,26\%), $29(25,2 \%)$ obesas tipo I, 10 $(8,6 \%)$ obesas tipo II y $9(9,7 \%)$ obesas tipo III.

La distribución de gestantes según el IMC y la edad, se puede observar en la tabla 1 . No hubo adolescentes

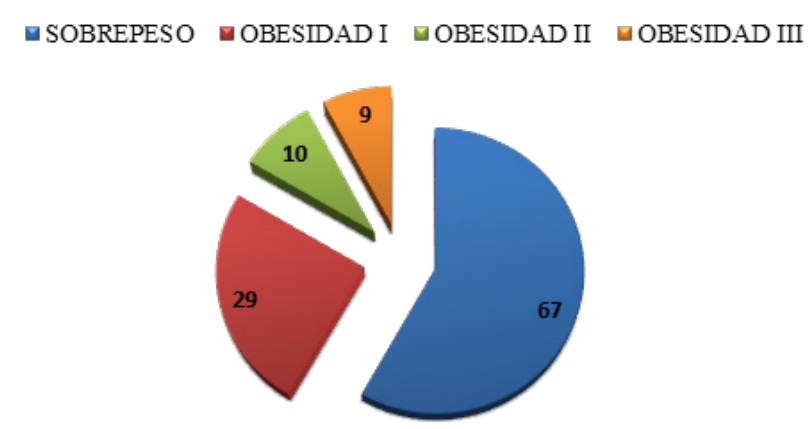

Gráfico 1. Frecuencia de sobrepeso/obesidad 
Tabla 1. Edad materna según el índice de masa corporal

\begin{tabular}{lcccc}
\hline $\begin{array}{l}\text { Edad } \\
\text { en }\end{array}$ & $\begin{array}{c}\text { Sobrepeso } \\
\mathrm{n}(\%) \\
\mathrm{n}=67\end{array}$ & $\begin{array}{c}\text { Obesidad I } \\
\mathrm{n}(\%) \\
\mathrm{n}=29\end{array}$ & $\begin{array}{c}\text { Obesidad II } \\
\mathrm{n}(\%) \\
\mathrm{n}=10\end{array}$ & $\begin{array}{c}\text { Obesidad III } \\
\mathrm{n}(\%) \\
\mathrm{n}=9\end{array}$ \\
\hline $16-20$ & $7(10,4)$ & $1(3,4)$ & 0 & 0 \\
$21-25$ & $18(26,9)$ & $8(27,6 \%)$ & $3(30)$ & $1(11,1)$ \\
$26-30$ & $22(32,8)$ & $10(34,5)$ & $3(30)$ & $3(33,3)$ \\
$31-35$ & $15(22,4)$ & $7(24,1)$ & $3(30)$ & $3(33,3)$ \\
$>36$ & $5(7,5)$ & $3(10,3)$ & $1(10)$ & $2(22,2)$ \\
\hline
\end{tabular}

precoces. Entre las 67 pacientes con sobrepeso, el mayor porcentaje tenía entre 26 y 30 años (22 casos/32,8\%), seguidas por el grupo de 21 a 25 años (18 pacientes/26,9\%) y luego el grupo de 31 a 35 años (15 gestantes/22,4 \%). La obesidad tipo I tuvo una distribución similar. La distribución por grupos etarios de las pacientes con obesidad II y III fue más homogénea.

Había $35(30,5 \%)$ primigestas, $35(30,5 \%)$ con dos embarazos, $20(17,4 \%)$ con 3 embarazos y $25(21,7)$ con 4 o más. Con relación a la distribución según el IMC, se obtuvo que 21 de las gestantes con sobrepeso eran primigestas, 25 cursaban su segundo embarazo, 10 el tercero y 11 tenían 4 o más embarazos. Entre las pacientes con obesidad I, 14 tenían 1 o 2 embarazos, 5 tenían 3 y 10 tenían 4 o más. En la tabla 2 se puede observar la distribución completa según el número de embarazo y el IMC.

Tabla 2. Número de embarazos según el índice de masa corporal

\begin{tabular}{lcccc}
\hline $\begin{array}{l}\text { Número } \\
\text { de } \\
\text { Gestas }\end{array}$ & $\begin{array}{c}\text { Sobrepeso } \\
\mathrm{n}(\%) \\
\mathrm{n}=67\end{array}$ & $\begin{array}{c}\text { Obesidad I } \\
\mathrm{n}(\%) \\
\mathrm{n}=29\end{array}$ & $\begin{array}{c}\text { Obesidad II } \\
\mathrm{n}(\%) \\
\mathrm{n}=10\end{array}$ & $\begin{array}{c}\text { Obesidad III } \\
\mathrm{n}(\%) \\
\mathrm{n}=9\end{array}$ \\
\hline 1 & $21(31,3)$ & $7(24,1)$ & $3(30)$ & $4(44,4)$ \\
2 & $25(37,3)$ & $7(24,1)$ & $2(20)$ & $1(11,1)$ \\
3 & $10(14,9)$ & $5(17,2)$ & $2(20)$ & $3(33,3)$ \\
$4 \mathrm{o}+$ & $11(16,4)$ & $10(34,5)$ & $3(30)$ & $1(11,1)$ \\
\hline
\end{tabular}

Dentro de los antecedentes quirúrgicos más frecuentes se encontró: $34(29,6 \%)$ cesáreas, de las cuales 22 (65\%) tenían sobrepeso, 8 (24\%) tenían obesidad tipo I, $2(1,7 \%)$ tenían obesidad tipo II y $2(1,7 \%)$ tenían obesidad tipo III. No se encontró en la historia el reporte de la razón de la indicación de la cesárea. Además, se reportó un caso de salpingectomía y uno de laparotomía en pacientes con sobrepeso.

Con relación a los antecedentes o complicaciones en embarazos previos, se observaron: cesáreas $34(29,56 \%)$, preeclampsia $11(9,56 \%)$, aborto habitual $7(6,08 \%)$, aborto 7 (6,08\%), eclampsia 3 $(2,60 \%)$, mortinatos $3(2,6 \%)$, situación transversa $2(1,7 \%)$, hipocinesia fetal, muerte neonatal precoz, desproporción feto-pélvica, amenaza parto pretérmino y embarazo ectópico $1(0,86 \%)$ caso cada uno. La distribución según el IMC materno se puede observar en el gráfico 2.

La distribución de las gestantes de acuerdo con la edad de gestación en el momento de su ingreso, según su condición de adiposidad se presenta en la tabla 3 : $12(10,43 \%)$ embarazos pretérmino (10,4\%), 102 $(88,7 \%)$ estaban a término y 1 paciente $(0,8 \%)$ presentó un embarazo cronológico prolongado. Cinco pacientes $(7,5 \%)$ con embarazo pretérmino, 61 $(91,0 \%)$ a término y $1(1,5 \%)$ postérmino, tenían sobrepeso. Entre las pacientes con obesidad I, 4 $(13,8 \%)$ tuvieron parto pretérmino y $25(86,2 \%)$ a término. Entre las obesas tipo II, 2 (20\%) eran embarazos pretérmino y $8(80 \%)$ a término y entre las obesas tipo II hubo $1(11,1 \%)$ pretérmino y 8 $(88,9 \%)$ a término.

El peso previo al embarazo y la ganancia ponderal en $\mathrm{Kg}$ discriminados según el grado de adiposidad, se describe en la tabla 4. Entre las pacientes con sobrepeso, se encontró que 53 (79,1\%) pesaban entre 58 y $82 \mathrm{Kg}$ antes del embarazo y una $(1,5 \%)$ pesaba entre 83 y 107. No se pudo obtener la información de las restantes 13 mujeres con sobrepeso al momento 


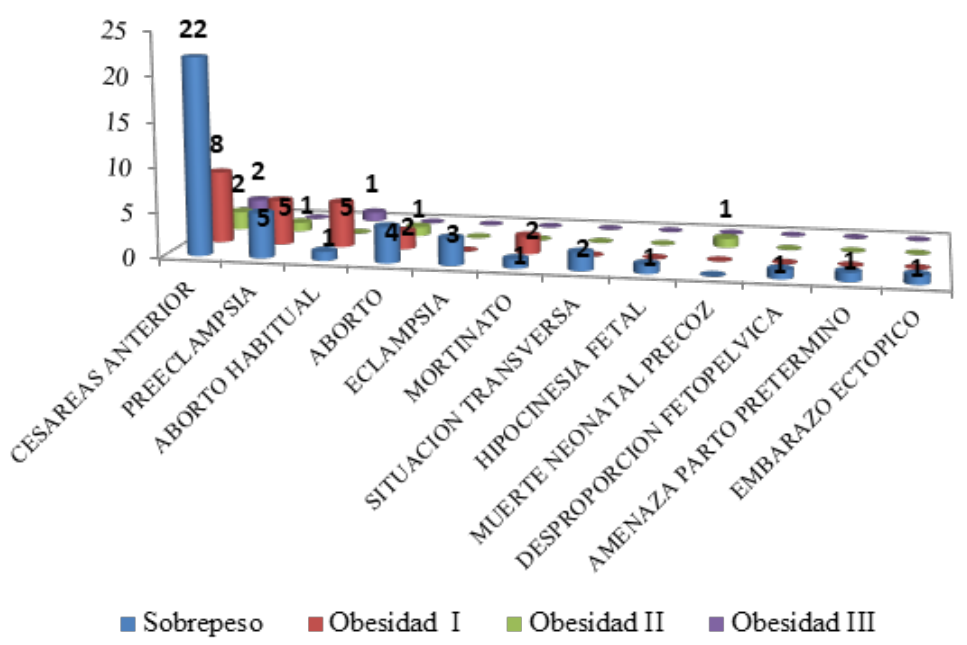

Gráfico 2. Distribución de los antecedentes según el índice de masa corporal

Tabla 3. Distribución de la edad gestacional según el índice de masa corporal

\begin{tabular}{lcccc}
\hline $\begin{array}{l}\text { Número } \\
\text { de }\end{array}$ & $\begin{array}{c}\text { Sobrepeso } \\
\mathrm{n}(\%) \\
\mathrm{n}=67\end{array}$ & $\begin{array}{c}\text { Obesidad I } \\
\mathrm{n}(\%) \\
\mathrm{n}=29\end{array}$ & $\begin{array}{c}\text { Obesidad II } \\
\mathrm{n}(\%) \\
\mathrm{n}=10\end{array}$ & $\begin{array}{c}\text { Obesidad III } \\
\mathrm{n}(\%) \\
\mathrm{n}=9\end{array}$ \\
\hline Pretérmino & $5(7,5)$ & $4(13,8)$ & $2(20)$ & $1(1,11)$ \\
A término & $61(91,0)$ & $25(82,2)$ & $8(80)$ & $8(88,9)$ \\
Postérmino & $1(1,5)$ & 0 & 0 & 0 \\
\hline
\end{tabular}

de la evaluación. Entre las gestantes con obesidad I, 6 $(20,7 \%)$ pesaban entre 58 y $82 \mathrm{Kg}, 15(51,7 \%)$ entre 83 y 107 y $1(3,4 \%)$ entre 108 y 132 . Se desconoce el peso previo en las restantes 7 pacientes. En las pacientes con obesidad II predominó el peso entre 83 y $107 \mathrm{Kg}(90 \%)$ y en las obesas III, el mayor grupo estaba entre108 y $132 \mathrm{Kg}(55,6 \%)$.

En cuanto a la ganancia de peso durante el embarazo, $85(73,9 \%)$ tuvieron un incremento menor a 10 kilos: 50 de las 67 que tenían sobrepeso $(74,6 \%), 22$ $(75,8 \%)$ de las que tenían obesidad tipo I, 6 (60\%) en el grupo de obesidad tipo II y $7(77,8 \%)$ en las obesas tipo III. La distribución se observa en la tabla 4. Se desconoce el dato en 13 pacientes con sobrepeso, 7 con obesidad I y 1 con obesidad III.
Tabla 4. Distribución de peso previo al embarazo y la ganancia de peso según el índice de masa corporal

\begin{tabular}{lcccc}
\hline $\begin{array}{l}\text { Peso } \\
(\mathrm{Kg}) *\end{array}$ & $\begin{array}{c}\text { Sobrepeso } \\
\mathrm{n}(\%) \\
\mathrm{n}=67\end{array}$ & $\begin{array}{c}\text { Obesidad I } \\
\mathrm{n}(\%) \\
\mathrm{n}=29\end{array}$ & $\begin{array}{c}\text { Obesidad II } \\
\mathrm{n}(\%) \\
\mathrm{n}=10\end{array}$ & $\begin{array}{c}\text { Obesidad III } \\
\mathrm{n}(\%) \\
\mathrm{n}=9\end{array}$ \\
\hline $\begin{array}{l}\text { Peso } \\
\text { previo }\end{array}$ & & & & \\
$58-82$ & $53(79,1)$ & $6(20,7)$ & $1(10)$ & 0 \\
$83-107$ & $1(1,5)$ & $15(51,7)$ & $9(90)$ & $2(22,2)$ \\
$108-132$ & --- & $1(3,4)$ & --- & $5(55,6)$ \\
$>132$ & ---- & --- & --- & $1(11,1)$ \\
$\begin{array}{l}\text { Ganancia } \\
\text { de peso }\end{array}$ & & & & \\
(Kg) & & & & \\
$0-5$ & $35(52,2)$ & $12(41,4)$ & 0 & $7(77,8)$ \\
$6-10$ & $15(22,4)$ & $10(34,5)$ & $6(60)$ & \\
$11-15$ & $4(6,0)$ & ---- & $3(30)$ & $1(1,11)$ \\
$>15$ & ---- & --- & $1(10)$ & - \\
\hline
\end{tabular}

* Se desconoce el dato en 13 pacientes con sobrepeso, 7 con obesidad tipo I y 1 con obesidad tipo III

Con respecto a la vía del parto, su resolución más frecuente fue la vaginal: $70(60,9 \%)$. Se realizaron $45(38,3 \%)$ cesáreas. No aparecen reportadas las 
indicaciones de la solicitud de la intervención dentro de la hoja de protocolo quirúrgico. La distribución según el IMC se presenta en la tabla 5. Entre las 67 pacientes con sobrepeso, 47 (70 \%) tuvieron parto vaginal. Puede observarse que la frecuencia de parto vaginal se redujo a medida que aumenta el IMC.

Las complicaciones que se presentaron durante el embarazo según el IMC, se presentan en la tabla 6. Entre las pacientes con sobrepeso hubo $17(25,4 \%)$ infecciones vaginales, $10 \quad(14,9 \%)$ infecciones urinarias, $10(14,9 \%)$ casos de anemia leve o moderada y $4(6,0 \%)$ casos sin complicaciones. Entre las pacientes con obesidad I, predominaron las infecciones vaginales y los trastornos hipertensivos del embarazo

Tabla 5. Distribución de pacientes según la vía del parto y el índice de masa corporal

\begin{tabular}{|c|c|c|c|c|}
\hline $\begin{array}{l}\text { Número } \\
\text { de } \\
\text { Gestas }\end{array}$ & $\begin{array}{c}\text { Sobrepeso } \\
n(\%) \\
n=67\end{array}$ & $\begin{array}{c}\text { Obesidad I } \\
\text { n }(\%) \\
n=29\end{array}$ & $\begin{array}{c}\text { Obesidad II } \\
n(\%) \\
n=10\end{array}$ & $\begin{array}{c}\text { Obesidad III } \\
\text { n }(\%) \\
n=9\end{array}$ \\
\hline Parto & $47(70,1)$ & $15(51,7)$ & $4(40)$ & $4(44,4)$ \\
\hline Cesárea & $20(29,8)$ & $14(48,3)$ & $6(60)$ & $5(55,6)$ \\
\hline
\end{tabular}

(THE), con 7 casos $(24,1 \%)$ cada uno. Hubo 5 casos (50\%) de THE entre las obesas tipo II, y 5 casos $(55,6 \%)$ entre las obesas tipo III. Hubo tres pacientes entre las obesas tipo II y 2 pacientes entre las obesas tipo III, que presentaron más de una complicación.

Entre las complicaciones puerperales más frecuentes, se observó: ingresos a la unidad de cuidados intensivos (UCI) 8 (7\%), preeclampsia grave $5(4,3 \%)$, anemia grave $2(1,7 \%)$ y con 1 $(0,8 \%)$ casos, hemorragias, dehiscencia de herida, shock hipovolémico, rotura uterina y eclampsia. En el gráfico 3 se observa que las pacientes con sobrepeso presentaron: ingreso a UCI: dos casos, anemia: dos casos, hemorragia posparto, dehiscencia de la herida, shock hipovolémico, rotura uterina y preeclampsia grave: un caso cada una. Tanto entre las pacientes con obesidad I como en las tipo II, hubo un ingreso a UCI y una preeclampsia grave. Finalmente, entre las obesas tipo III, 4 ingresaron a UCI, dos tuvieron preeclampsia grave y un caso de eclampsia.

Entre las complicaciones neonatales se observaron: 12 casos de bajo peso (10,4\%), 29 (7,8 \%) prematuridad,

Tabla 6. Distribución según las complicaciones obstétricas y el índice de masa corporal

\begin{tabular}{|c|c|c|c|c|}
\hline Complicaciones & $\begin{array}{c}\text { Sobrepeso } \\
n(\%) \\
n=67\end{array}$ & $\begin{array}{c}\text { Obesidad I } \\
\mathrm{n}(\%) \\
\mathrm{n}=29\end{array}$ & $\begin{array}{c}\text { Obesidad II } \\
n(\%) \\
n=10\end{array}$ & $\begin{array}{c}\text { Obesidad III } \\
n(\%) \\
n=9\end{array}$ \\
\hline Infecciones vaginales & $17(25,4)$ & $7(24,1)$ & $2(20)$ & $1(11,1)$ \\
\hline Infecciones urinarias & $10(14,9)$ & $2(6,9)$ & $1(10)$ & --- \\
\hline Anemia & $10(14,9)$ & $1(3,4)$ & --- & $1(11,1)$ \\
\hline Ruptura prematura de membranas & $8(11,9)$ & $3(10,3)$ & $1(10)$ & --- \\
\hline Distocia de presentación & $6(9,0)$ & $2(6,9)$ & $1(10)$ & $3(33,3)$ \\
\hline Trastornos hipertensivos del embarazo & $3(4,5)$ & $7(24,1)$ & $5(50)$ & $5(55,6)$ \\
\hline Otras & $9(13,4)$ & $7(24,1)$ & $3(30)$ & $1(11,1)$ \\
\hline
\end{tabular}




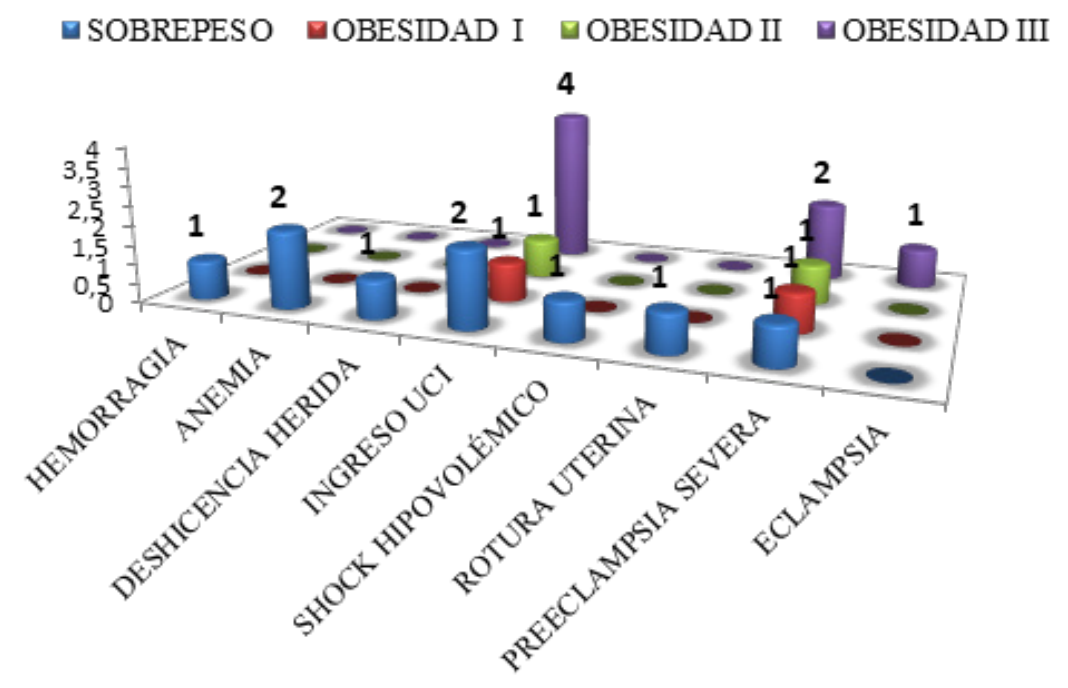

Gráfico 3. Complicaciones puerperales según el índice de masa corporal

$4(3,4 \%)$ óbito fetal, $2(1,76 \%)$ restricción del crecimiento intrauterino (RCIU) y $1(0,8 \%)$ casos de neonatos con asfixia perinatal, síndrome de dificultad respiratoria y aspiración meconial, respectivamente. En el gráfico 4 se presentó la distribución según la adiposidad materna.

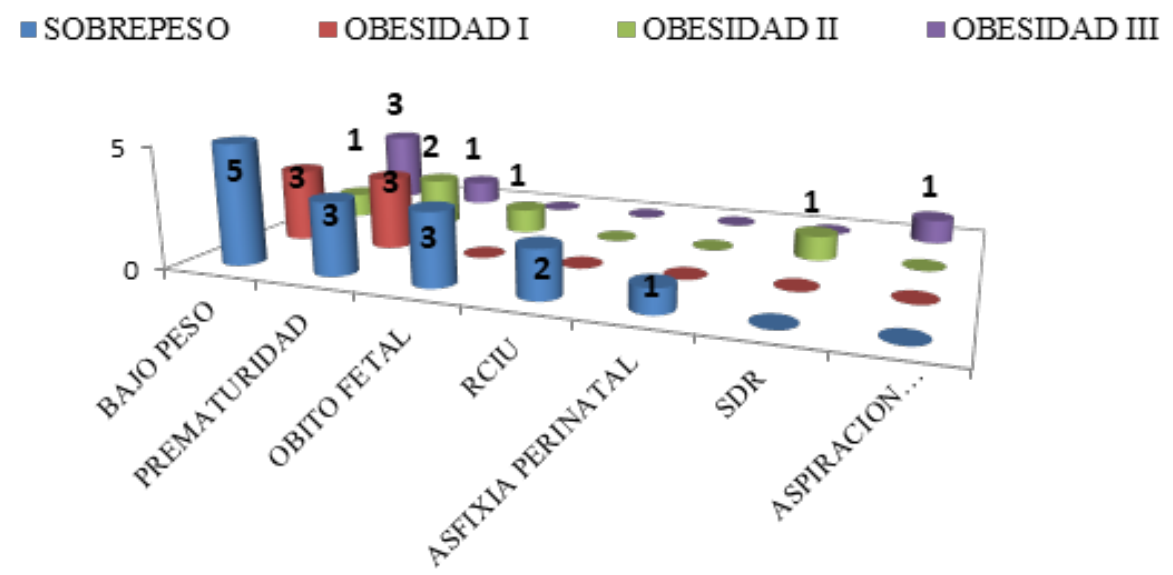

Gráfico 4. Complicaciones neonatales según el índice de masa corporal 


\section{DISCUSIÓN}

La adiposidad es un complejo síndrome clínico heterogéneo crónico, en el cual múltiples factores interactúan para producir un estado de balance energético positivo que lleva al aumento del peso corporal entre otras cosas. Existen diversos determinantes biológicos, ambientales, genéticos, cerebro-intestinales, determinantes prenatales, embarazo, discapacidad física, etc.

En los EE. UU., la obesidad en mujeres en edad reproductiva afecta el $31,8 \%$ de las mujeres y aumenta al 58,5\% cuando se combina con el sobrepeso, con tendencia cada vez más al aumento progresivo en el tiempo, al igual que en Europa (9). El embarazo de mujeres con obesidad es un conflicto para la salud pública de cualquier país, pues incrementa los riesgos obstétricos y neonatales. Según la OMS, en 2014, 1900 millones de adultos de 18 o más años tenían sobrepeso, de los cuales más de 600 millones eran obesos; $40 \%$ eran mujeres con sobrepeso y $15 \%$ eran obesas (17). En la presente investigación se evaluaron 115 pacientes con sobrepeso y obesidad, es decir, con IMC superior a $25 \mathrm{Kg} / \mathrm{m}^{2}$. No se estableció la frecuencia general porque todas las pacientes incluidas tenían algún grado de sobrepeso. Sin embargo, a pesar de que predominaron los casos de sobrepeso y obesidad tipo I, hubo $16,5 \%$ de pacientes con obesidad II y III. En América Latina, se describió en un estudio que 37,1\% tenían sobrepeso, $11,9 \%$ obesidad tipo I, 0,6\% obesidad tipo II y $0,3 \%$ obesidad tipo III o mórbida (18). Previamente, en 2015, Cajas (19) encontró que 80 (50\%) de sus casos estaban en obesidad I, 67 (42\%) obesidad tipo II y solo $8 \%$ con obesidad extrema, con un índice de masa corporal mayor de $40 \mathrm{~kg} /$ $\mathrm{m}^{2}$. Obviamente, a medida que avanza la intensidad de la obesidad, disminuye la frecuencia absoluta de casos, concentrándose en las primeras categorías de adiposidad como son el sobrepeso y obesidad I, lo que coincide con los hallazgos de esta investigación.
No existen muchos estudios basados en evidencia que especifiquen realmente cuál es la incidencia real de sobrepeso y obesidad en la gestante, sin embargo, en un estudio realizado en Paraguay, entre gestantes que asistían a control prenatal se encontró que una de cada 12 mujeres estudiadas presentaba algún grado de obesidad (20,21). El sobrepeso ha aumentado en la mujer en edad fértil en los últimos 30 años, según el Instituto Nacional Mexicano de Perinatología, se observa un porcentaje mayor del $80 \%$ de gestantes con sobrepeso y obesidad (22).

La OMS reconoce la obesidad como un problema de salud pública a escala mundial y algunos autores la han considerado como una pandemia del siglo XXI (23) de la que no se escapa la población obstétrica, por ello la razón de múltiples estudios avocados a determinar los efectos maternos y perinatales que pocas veces estiman la incidencia de madres con esta comorbilidad.

Para el año 2003, Moreno y cols. (24) realizaron un estudio en Lima, Perú, para evaluar la obesidad pregestacional como factor de riesgo asociado a preeclampsia. Reportaron una media de 26,9 años de edad para el grupo estudio y 26,1 para el grupo control. Según la encuesta realizada por el Sistema de Salud de Chile (2009-2010), demostró que el 51\% de las mujeres entre 15 y 44 años tienen un IMC mayor a 25, es decir, más de la mitad de las mujeres chilenas en edad fértil presenta sobrepeso u obesidad $(21,25)$. Borrero y cols. (26), en 2011, estudiando las complicaciones maternas y perinatales de la obesidad en Colombia, encontraron que el $23 \%$ de sus gestantes eran menores de 25 años, $69 \%$ entre 25 y 34 años, criterio que fue compartido por Piedra (27) en la población de Cuenca-Ecuador, en el año 2011, quien reporta que la población joven fértil entre 20 y 39 años era del $50 \%$. En este estudio se encontró una asociación similar, de ahí que el sobrepeso y la obesidad representan un desafío en el control del embarazo y la resolución del parto. 
Muy pocos estudios evalúan la variable edad de gestación como interviniente en el resultado de la gestación en pacientes con sobrepeso y obesidad. En Venezuela, enun estudiorealizado en el HospitalCentral de Maracay, estado Aragua, se encontró que la edad media promedio de gestación fue 35,7 $\pm 5,03$ semanas con un porcentaje de $6,6 \%$ de partos pretérmino, similar a lo hallado en esta investigación (14), hecho que también concuerda con lo reportado por el estudio sobre obesidad y riesgo de parto pretérmino, realizado por Cnattingius y cols. (28), en el cual de 1599551 partos, 3082 eran extremadamente prematuros, 68993 muy prematuros y 67059 moderadamente prematuros, proporcional con el aumento del cociente del IMC de forma exponencial. La prematuridad somete a los productos de la gestación a mayor morbilidad, discapacidad a largo plazo e incluso mortalidad.

Un estudio de casos y controles realizado en Landspitali University Hospital, en Islandia, entre 1989-2004, donde se comparó a mujeres con peso normal y mujeres con sobrepeso u obesidad, observaron desórdenes hipertensivos elevados en las embarazadas del último grupo con 1,63 veces el riesgo de enfermedad hipertensiva gestacional en las embarazadas con sobrepeso y 2,79 veces de riesgo en las obesas (29). La obesidad se asocia con mucha frecuencia a la hipertensión gestacional y se ve más elevada aun cuando se relaciona con edades avanzadas durante el embarazo y el incremento de IMC $>25$ $\mathrm{kg} / \mathrm{m}^{2}$ aumenta la prevalencia de hipertensión en mujeres en edad reproductiva $(22,30)$. Las mujeres que han presentado eclampsia e hipertensión arterial gestacional recurrente en embarazos subsiguientes tienen mucha más probabilidad de desarrollar hipertensión arterial crónica (24). En la presente serie, se observó el antecedente de THE en 14 casos, 8 de los cuales (57\%) ocurrieron en pacientes con sobrepeso y 6 en pacientes con obesidad (42,8\%). El riesgo de hipertensión gestacional se encuentra 2,5 veces incrementado en mujeres obesas cursando su primer embarazo y 3,2 en aquellas con obesidad mórbida, 1,6 veces mayor riesgo de preeclampsia en pacientes obesas y 3,3 en obesas graves. En un estudio general se observó que por cada $5-7 \mathrm{~kg} / \mathrm{m}^{2}$ se dobla el riesgo de preeclampsia y el riesgo de hipertensión crónica aumenta hasta 5 veces en aquellas mujeres con sobrepeso $(21,31,32)$

En las pacientes evaluadas en este estudio, entre los 20 casos de THE, 4,5\% ocurrió en pacientes con sobrepeso, $24 \%$ en mujeres con obesidad tipo I, $50 \%$ con obesidad II y 55,6 \% con obesidad III. Se observa claramente como la frecuencia aumenta a medida que aumenta el IMC.

Aunque no son tan frecuentes como la diabetes gestacional o el THE, el sobrepeso y la obesidad materna están asociados a múltiples complicaciones como aborto espontáneo, parto pretérmino, muerte fetal intrauterina, macrosomía fetal, alteraciones del trabajo de parto y mayor tasa de cesáreas. Un aumento significativo de infección de la herida quirúrgica en la poscesárea se observa en mujeres con sobrepeso y el riesgo se concentra entre las pacientes obesas clase III (con un IMC $>45 \mathrm{~kg} / \mathrm{m}^{2}$ ), según lo señalan Lozano y cols. (21). Otros estudios revelan datos sobre prevalencia aumentada de infección de vías urinarias, vaginitis e infecciones por estreptococos en pacientes obesas. La obesidad materna además produce sobre crecimiento placentario que llevaría a hipoxia fetal, también está asociada con un aumento en la incidencia de trombosis fetal (21). Los resultados de esta investigación muestran una frecuencia elevada de infecciones vaginales, infecciones urinarias y anemia, entre otras patologías, sin embargo, solo se observó relación directa con el IMC en los casos de THE.

Yépezy cols. (33), entre agosto 2008 y septiembre 2009, realizaron un estudio en la Maternidad Concepción Palacios, Caracas, para evaluar la asociación entre el síndrome metabólico y las complicaciones maternas, 
fetales y neonatales. Encontraron 34,2 \% presentó trastornos hipertensivos del embarazo, 23,7 \% de diabetes, $26,3 \%$ de parto pretérmino y $2,6 \%$ de infección puerperal, parecido a lo encontrado en el estudio de sobrepeso y obesidad pregestacional como factor de riesgo de cesárea y complicaciones perinatales desarrollado De La Calle y cols. (34) quienes señalan que la diabetes gestacional fue más frecuente en las gestantes con sobrepeso y obesidad pregestacional que en las de peso normal. Con relación a este punto, en esta investigación no se documentó el antecedente de diabetes pregestacional ni el diagnostico de diabetes en el embarazo actual, pero sí se observó una alta frecuencia de pacientes con peso elevado antes del embarazo. Asimismo, la ganancia ponderal durante el embarazo fue mayor entre aquellas con obesidad tipo II.

Con relación a las complicaciones neonatales, Buele y cols. (35) observaron que los recién nacidos que tuvieron algún tipo de patología en el momento del nacimiento fueron productos de madres que se encontraban con obesidad (66,7 \%) de todos los casos. Además, 33,3\% de los recién nacidos de madres con sobrepeso y obesidad presentaron macrosomía fetal. En 2017, Vilches (36) reportó que el $31 \%$ de las patologías presentes al término del embarazo, se debieron a alteración en el crecimiento fetal intrauterino, ya sea por exceso (grandes para edad gestacional: 16,9 \%) o por déficit (restricción de crecimiento intrauterino: 14,1\%).

En el presente estudio, los hijos de madres con sobrepeso $\mathrm{u}$ obesidad presentaron prematuridad, bajo peso, asfixia perinatal, síndrome de dificultad respiratoria y aspiración meconial, sin embargo, la frecuencia no parece ser mayor que la de la población general. Es importante acotar que estas complicaciones traen consecuencia en los primeros años de vida e incluso en la vida adulta. Los recién nacidos con peso bajo o elevado para la edad gestacional presentan una mayor susceptibilidad a desarrollar obesidad en la vida extrauterina (37).
La sociedad tiene como mito que en el embarazo la mujer "tiene que comer por dos" lo que conlleva a un mayor consumo proteínico-calórico, convirtiéndose en una alimentación incontrolada. Según Lozano y cols. (21), la Academia Nacional de Ciencias y el Instituto de Medicina (IOM) de los Estados Unidos recomiendan que las mujeres que comienzan el embarazo con sobrepeso, con un IMC de 26-29, deban ganar entre 15-25 libras y las mujeres obesas con un IMC de 30 o más antes del embarazo deben aspirar a una ganancia de peso de 15 libras, aunque se aconseja a las mujeres obesas limitar su ganancia de peso entre 5 a $9 \mathrm{~kg}$. Una ganancia de peso de $5 \mathrm{~kg}$ o menos puede reducir el número de complicaciones sin aumentar el número de resultados adversos.

El riesgo de complicaciones que corre la embarazada y su bebe si ella se encuentra con sobrepeso u obesidad en el momento de embarazarse es muy elevado, por tal razón, se debe hacer prevención tratando la adiposidad de la paciente antes del embarazo, para así reducir los riesgos y garantizar un embarazo con un feliz término.

Con base a los resultados se puede concluir:

1. En este grupo de gestantes, todas con algún grado de sobrepeso, la frecuencia de las distintas categorías fue: sobrepeso: $58,3 \%$, obesidad I: $25,2 \%$, obesidad II: $8,6 \%$ y obesidad III: de $9,7 \%$.

2. Los antecedentes presentes fueron la cesárea anterior, los trastornos hipertensivos del embarazo y el aborto.

3. Las complicaciones más frecuentes en el embarazo actual fueron las infecciones vaginales, urinarias, la anemia, y los trastornos hipertensivos del embarazo.

4. Entre las complicaciones neonatales predominó el bajo peso y la prematuridad, pero su frecuencia no fue mayor a la de la población general. 
Se recomienda:

Fortalecer medidas preventivas por medio de consulta por nutrición a todas las mujeres con sobrepeso $\mathrm{u}$ obesidad, y se les debe alentar a seguir un programa de ejercicios y asesoramiento nutricional acorde al índice de masa corporal, que deben continuar después del parto y antes de intentar un nuevo embarazo.

\section{REFERENCIAS}

1. Alberico S, Montico M, Barresi V, Monasta L, Businelli C, Soini V, et al.; Multicentre Study Group on Mode of Delivery in Friuli Venezia Giulia. The role of gestational diabetes, pre-pregnancy body mass index and gestational weight gain on the risk of newborn macrosomia: results from a prospective multicentre study. BMC Pregnancy Childbirth. 2014; 14:23. doi: 10.1186/1471-2393-14-23.

2. ACOG Practice Bulletin No 156: Obesity in Pregnancy. Obstet Gynecol. 2015; 126(6):e112-e126. doi: 10.1097/AOG.0000000000001211.

3. Organización Mundial de la Salud [Internet]. Ginebra: Obesidad y sobrepeso. Nota descriptiva $N^{\circ} 311$ [consultado 12 septiembre 2017]. Disponible en: https://www.who.int/es/news-room/fact-sheets/ detail/obesity-and-overweight

4. Quintana N, Rey D, Sisi T, Antonelli C, Ramos M. Preclampsia. Rev Posgrado VI Catedra Med [Internet]. 2003 [consultado 18 agosto 2018]; 133:16-20. Disponible en: https://med.unne.edu.ar/ revistas/revista133/preeclam.htm

5. Menéndez GE, Navas I, Hidalgo Y, Espert J. El embarazo y sus complicaciones en la madre adolescente. Rev Cubana Obstet Ginecol [Internet]. 2012 [consultado 20 de noviembre de 2017]; 38(3):333-342. Disponible en: https:// www.medigraphic.com/cgi-bin/new/resumen. cgi? IDARTICULO $=35125$

6. Kristensen J, Vestergaard M, Wisborg K, Kesmodel U, Secher NJ. Pre-pregnancy weight and the risk of stillbirth and neonatal death. BJOG. 2005; 112(4):403-408. doi: 10.1111/j.14710528.2005.00437.x.

7. Rosso P. A new chart to monitor weight gain during pregnancy. Am J Clin Nutr. 1985; 41(3):644-652. doi: 10.1093/ajen/41.3.644.
8. Ruhstaller K. Induction of labor in the obese patient. Semin Perinatol. 2015; 39(6):437-440. doi: 10.1053/j.semperi.2015.07.003.

9. O'Reilly JR, Reynolds RM. The risk of maternal obesity to the long-term health of the offspring. Clin Endocrinol (Oxf). 2013; 78(1):9-16. doi: 10.1111/ cen. 12055 .

10. Abenhaim HA, Kinch RA, Morin L, Benjamin A, Usher R. Effect of prepregnancy body mass index categories on obstetrical and neonatal outcomes. Arch Gynecol Obstet. 2007; 275(1):39-43. doi: 10.1007/s00404-006-0219-y.

11. Campoy C, Martín-Bautista E, García-Valdés L, Florido J, Agil A, Lorente JA. et al. Estudio de la influencia de la nutrición y genética maternas sobre la programación del desarrollo del tejido adiposo fetal: Estudio PREOBE. Nutr Hosp [Internet]. 2008 [consultado 23 de octubre de 2018]; 23(6):584-590. Disponible en: http://scielo. isciii.es/scielo.php?script $=$ sci_arttext\&pid $=$ S0212$16112008000800010 \& \operatorname{lng}=$ es.

12. Organización Mundial de la Salud [Internet]. Ginebra: Declaración de la OMS sobre tasas de cesárea. Resumen ejecutivo; 2015 [consultado 19 de octubre de 2018]. Disponible en: https://www. who.int/reproductivehealth/publications/maternal_ perinatal_health/cs-statement/es/

13. Febres $\bar{F}$, Zimmer E, Guerra C, Zimmer E, Gil J. Nuevos conceptos en diabetes mellitus gestacional: evaluación prospectiva de 3070 mujeres embarazadas. Rev Obstet Ginecol Venez [Internet]. 2000 [consultado 1 de octubre de 2018] 60(4):229-237. Disponible en: http://www.sogvzla. org.ve/sogvzla20186/cms/svcobtenerpdfrevista. php? $\mathrm{id}=0000000051 \&$ tipo $=$ normal $\&$ fila $=4$

14. Torres D, Sandoval F. Obesidad y sus complicaciones en gestantes atendidas en la emergencia obstétrica del servicio autónomo Hospital Central de Maracay enero-julio 2014 [Tesis en Internet]. Valencia (Carabobo): Universidad de Carabobo; 2014 [consultado 1 de octubre de 2018]. Disponible en: http://mriuc.bc.uc.edu.ve/bitstream/ handle/123456789/1265/Dtorres? sequence $=1$

15. Zimmet P. Nueva definición mundial del síndrome metabólico propuesta por la Federación Internacional de Diabetes: fundamento y resultados. Rev Esp Cardiol [Internet]. 2005 [consultado 13 de octubre de 2018]; 58:1371-1376. Disponible en: https://www. revespcardiol.org/index.php? $\mathrm{p}=$ revista\&tipo $=$ pdf- 
simple\&pii $=13082533$

16. Catalano PM. Obesity, insulin resistance, and pregnancy outcome. Reproduction. 2010; 140(3):365-371. doi: 10.1530/REP-10-0088.

17. Pizzi R, Fung L. Obesidad y mujer. Rev Obstet Ginecol Venez [Internet]. 2015 [consultado 25 de septiembre de 2018]; 75(4): Disponible en: http://www.sogvzla. org.ve/sogvzla $20186 / \mathrm{cms} / \mathrm{svcobtenerpdfrevista.}$ php?id $=0000000078 \&$ tipo $=$ normal $\&$ fila $=2$

18. Benllochpiquer EH. Excesivo peso pregestacional Vs complicaciones maternas y neonatales en el Instituto Nacional Materno Perinatal, 2015 [Tesis en internet]. Lima: Universidad Nacional Mayor de San Marcos; 2015 [consultado 12 de noviembre de 2020]. Disponible en: https://cybertesis.unmsm.edu. pe/handle/20.500.12672/6406

19. Cajas G. Obesidad materna y complicaciones materno-fetales [Tesis en Internet]. Guatemala: Universidad de San Carlos de Guatemala; 2015 [consultado: 12 de noviembre de 2020]. Disponible en: https://core.ac.uk/download/pdf/35292756.pdf

20. Mendoza L, Pérez B, Sánchez S. Estado nutricional de embarazadas en el último mes de gestación y su asociación con las medidas antropométricas de sus recién nacidos. Pediatr [Internet]. 2010 [consultado 12 de octubre de 2018]; 37(2): 91-96. Disponible en: https://dialnet.unirioja.es/servlet/ articulo? codigo $=4800205$

21. Lozano A, Betancourth W, Turcios LJ, Cueva JE, Ocampo D, Portillo CV, et al. Sobrepeso y obesidad en el embarazo. Complicaciones y manejo. Arch Med. 2016; 12(3):11-18. doi: 10.3823/1310

22. Sánchez BJ, Sámano R, Pinzón I, Balas M, Perichart O. Factores socioculturales y del entorno que influyen en las prácticas alimentarias de gestantes con y sin obesidad. Rev Salus Pública Nutr [Internet]. 2010 [consultado 16 de febrero de 2018]; 11(2):1-11. Disponible en: https://www.medigraphic.com/cgibin/new/resumen.cgi?IDARTICULO $=25926$

23. Ceballos-Macías JJ, Pérez Negrón-Juárez R, FloresReal JA, Vargas-Sánchez J, Ortega-Gutiérrez G, Madriz-Prado R, et al. Obesidad. Pandemia del siglo XXI. Rev Sanid Mil [Internet]. 2019 [consultado 11 de abril de 2018]; 72(5-6):332-338. Disponible en: http://www.scielo.org.mx/scielo.php?script=sci arttext\&pid=S0301-696X2018000400332\&lng=es.

24. Moreno Z, Sánchez S, Piña F, Reyes A, William M. Obesidad pregestacional como factor de riesgo asociado a preeclampsia. An Fac Med [Internet].
2003 [consultado 10 de septiembre de 2018]; 64(2):101-106. Disponible en: http://www.scielo. org.pe/scielo.php? script $=$ sci_arttext\&pid $=\mathrm{S} 1025$ 55832003000200004\&lng=es.

25. Farías. Obesidad materna: severo problema de salud pública en Chile. Rev Chil Obstet Ginecol. 2013; 78(6): 409 - 412. doi:10.4067/S071775262013000600001

26. Borrero SP, Cubillos AP. Complicaciones maternas y perinatales de la obesidad en mujeres atendidas en el Hospital Universitario Hernando Moncaleano Perdomo de Neiva durante el 2011 [Tesis en Internet]. Neiva: Universidad Surcolombiana; 2013 [consultado 23 de septiembre de 2018]. Disponible en: http://repositoriousco.co:8080/jspui/ bitstream/123456789/1368/1/TH\%20EGO\%20 0017.pdf

27. Piedra MA. Prevalencia de sobrepeso y obesidad, y su asociación con complicaciones obstétricas y perinatales, en gestantes con parto vaginal o cesárea, Hospital Vicente Corral Moscoso Cuenca 2012. Cuenca (Ecuador): Universidad de Cuenca; 2013 [consultado 25 de septiembre de 2018]. Disponible en: http://dspace.ucuenca.edu.ec/ bitstream/123456789/4010/1/MEDGO43.pdf

28. Cnattingius S, Villamor E, Johansson S, Edstedt Bonamy AK, Persson $\mathrm{M}$, Wikström AK, et al. Maternal obesity and risk of preterm delivery. JAMA. 2013; 309(22):2362-2370. doi: 10.1001/ jama.2013.6295.

29. Gudnadóttir TA, Bateman BT, Hernádez-Díaz S, Luque-Fernandez MA, Valdimarsdottir U, Zoega H. Body Mass Index, Smoking and Hypertensive Disorders during Pregnancy: A Population Based Case-Control Study. PLoS One. 2016; 11(3):e0152187. doi: 10.1371/journal. pone. 0152187.

30. Davies G, Maxwell C, McLeod L. Obesity in pregnancy. JOGC. 2018; 40(8):630-639. DOI: https://doi.org/10.1016/j.jogc.2018.05.018

31. Poll JA, Rueda NM, Ramos L, Poll A, Campos I. Riesgo cardiovascular e hipertensión arterial crónica en embarazadas. MEDISAN [Internet]. 2013 [consultado 27 de septiembre de 2018]; 17(2):213-220. Disponible en: http://scielo.sld. cu/scielo.php?script=sci_arttext\&pid=S102930192013000200007\&lng=es.

32. Barrera G, Germain A. Obesidad y embarazo. Rev Méd Clín Condes [Internet]. 2012 [consultado 28 
de septiembre de 2018]; 23(2):154-158. Disponible

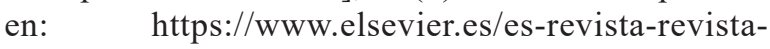
medica-clinica-las-condes-202-articulo-obesidadembarazo-S0716864012702924

33. Yépez MC, Zeppenfel ME, Colón JA, Zimmer E. Síndrome metabólico durante el embarazo. Complicaciones materno-fetales. Rev Obstet Ginecol Venez [Internet]. 2011 [consultado 24 de septiembre de 2018]; 71(2):77-87. Disponible en: http://www.sogvzla.org.ve/ sogvzla $20186 / \mathrm{cms} / \mathrm{svcobtenerpdfrevista.}$ php?id $=0000000008 \&$ tipo $=$ normal $\&$ fila $=3$

34. De La Calle M, Armijo O, Martín E, Sancha M, Magdaleno F, Omeñaca $\mathrm{F}$, et al. Sobrepeso y obesidad pregestacional como factor de riesgo de cesárea y complicaciones perinatales. Rev Chil Obstet Ginecol. 2009; 74(4): 233-238. http://dx.doi. org/10.4067/S0717-75262009000400005

35. Buele LC, Ordoñez SR. Incidencia de sobrepeso y obesidad materna y su relación con los principales riesgos obstétricos en mujeres gestantes atendidas en el área de Ginecología del Hospital cantonal de Alamor durante el periodo octubre 2011- julio 2012
[Tesis en internet]. Loja (Ecuador): Universidad Católica de Loja; 2013 [consultado 15 de diciembre de 2018]. Disponible en: http://dspace.utpl.edu.ec/ bitstream/123456789/6596/1/Tesis\%20.pdf

36. Vilches Sáez FN. Resultados maternos y perinatales en gestantes con sobrepeso y obesidad del Hospital Clínico Herminda Martin, Chillán. Concepción (Chile): Universidad de Concepción; 2017 [consultado 25 de septiembre de 2018]. Disponible en: http://repositorio.udec.cl/bitstream/11594/2668/4/ Tesis_resultados_maternos_y_perinatales.pdf

37. Toledo J, Cubillos G, Gómez O. Asociación entre obesidad e infecciones: un estudio de corte transversal. Rev Med [Internet]. 2014 [consultado 26 de septiembre de 2018]; 22(1):28-34. Disponible en: https://www.redalyc.org/pdf/910/91032438004. pdf
Recibido 26 de febrero de 2021 Aprobado 20 de mayo de 2021 